\title{
Blockchain and genomic research legal regulation: element in mechanism for interest's balancing
}

\author{
Aleksey Kubyshkin ${ }^{1, *}$, Daria Ponomareva $^{2}$ \\ ${ }^{1}$ Advocate's Office "SanctaLex" of the Moscow City Bar Association, Moscow, Russia \\ ${ }^{2}$ Kutafin Moscow State Law University (MSAL), Department of Legal Practice, Moscow, Russia
}

\begin{abstract}
The article analyzes blockchain technology in relation to the field of legal regulation of genomic research. The key features of this technology, which allow one to consider it as one of the elements of the mechanism for ensuring the balance of private, group and public interests are highlighted. Examples of the use of blockchain technology in healthcare are given, and proposals of various researchers on the use of this technology in the field of genomic research are analyzed. The analysis and classification of interests in the considered sphere of social relations is carried out, factors that must be taken into account when ensuring the balance of interests are given. Authors propose an approach to the mechanism of ensuring the balance, which, according to the authors, consist of three elements. At the same time, the place of blockchain technology in the specified mechanism is determined, conclusions and approaches are proposed for using this technology to ensure a balance of private, group and public interests.
\end{abstract}

\section{Introduction}

The modern world is characterized by significant breakthroughs in various fields of knowledge, primarily in the field of genomic research. In addition, it is generally accepted that the modern world differs significantly from the former, including in the economic way, which allows us to talk about modern society as information or post-industrial one.

Information society is a society in which information and the level of its application and accessibility radically affect the economic and socio-cultural living conditions of citizens [1].

The information society is opposed to the industrial society, formed in the process and as a result of industrialization, the development of machine production, the emergence of adequate forms of labor organization, the application of the achievements of technical and technological progress [2].

According to American sociologist D. Bell, the information society is a post-industrial society characterized by the transition from the production of things to the production of services, changes in employment and the central role of theoretical knowledge [3].

With regard to the post-industrial, information society, we can also talk about the information economy, in which the productivity and competitiveness of factors or agents (be it an individual, a firm or a national economy) depends on the ability to generate, process and effectively use information based on knowledge [4].

According to a number of scientists, a characteristic feature of the information economy is the formation of so-called network structures, network relations. The process of formation of the information economy changes the business environment and the dynamics of competition for all subjects of economic activity. Network structures and forms of management organize a space of close interaction between various spheres (technological, political, social, cultural) [5].

\section{Blockchain technology. Definition, characteristics}

One of the brightest technological manifestations of recent times is information technology blockchain, the so-called distributed ledger technology.

Blockchain is a distributed database consisting of a "chain of blocks", block storage devices are not connected to a common server, the database allows one to control the reliability of transactions without supervision of any financial regulators. Blockchain is a distributed and decentralized database formed by participants, in which it is impossible to falsify data due to the chronological record and public confirmation of the transaction by all network participants [6].

Initially, this technology was used for the formation and circulation of so-called cryptocurrencies. Nevertheless, this technology has a number of characteristics that make it possible to use it not only in the financial sphere, but also in other areas of social life such as notaries, cadasters, registers of property rights, taxes and benefits, violations and fines, user identification, registration of acts, civil status, public services, healthcare, education, art transfer, supplies, fair voting systems, loyalty programs, lotteries, etc. [7].

Corresponding author: a.kubyshkin@gmail.com 
Various authors cite the main characteristics of blockchain technology, which may be important in relation to the use of this technology in the mechanism of legal regulation of genomic research.

The most important aspects of blockchain technology are: (1) decentralization (i.e., one object cannot manage the database), (2) immutability (i.e., no previous record can be changed) and (3) security (i.e., accounts are protected by advanced cryptographic methods) [8].

Blockchain is a publicly distributed ledger that seals blocks with timestamps and encrypted hash links in a secure and immutable manner [9]. Blockchain uses timestamp technology to achieve data traceability and verifiability, which means that it offers a secure and transparent method to track the ownership of assets before, during, and after any transaction [10]. It enables transaction processes without the need for a trusted third party because of its traceability, smart contracts, resistance to tampering, decentralization, and encryption algorithm [11]. The open-source sharing protocol embedded into blockchain enables simultaneous data logging and storage by all participants, which ensures that the details of the recorded transactions cannot be retroactively changed without the full agreement of the network [12]. The architecture and governance are decentralized, which makes it highly fault-tolerant and resistant to attacks [13].

Russian scientists also note that the main characteristics of this technology that determine its attractiveness for use in various spheres of social relations are:

1) decentralization;

2) anonymity;

3) autonomy;

4) the use of cryptography;

5) adding special time stamps to each transaction [7].

The characteristics allow the use of blockchain technology in many areas of social relations.

\section{The usage of blockchain technology}

For quite some time this technology has been used for instance in health care. Currently, a number of projects based on the use of this technology are known, which have been successfully implemented and work in real life.

For example, Estonia became the first country to implement blockchain on a national scale. The eHealth Foundation of Estonia has been operating since 2005. In 2016, the eHealth Foundation teamed up with Guardtime, a data security company. Guardtime helped the foundation implement KSI (Keyless signature infrastructure), a blockchain technology that provides large-scale data authentication without relying on a centralized trusted authority. The project now contains over 1 million patient records and data. The KSI infrastructure ensures high security of medical data, their safety and integrity [14].

The government of Mongolia, together with FarmaTrust, launched a project to implement a drug tracking system based on blockchain technology, aimed at eliminating drug counterfeiting in the country [15].

The Novgorod Region of the Russian Federation became a pilot region in which, for the first time in Russia, Vnesheconombank (VEB) launched a program for monitoring drug circulation in a hospital using a blockchain system. This method of accounting will help to fight the falsification and leakage of expensive prescription drugs bought with budget money [16].

The use of blockchain technology is also possible in clinical trials in order to ensure the credibility, reliability of the results and to achieve other goals. Currently, the development of an information system for clinical trials based on blockchain technology is underway by a consortium, which includes the following Russian organizations: National Medical Research Center of Oncology named after N.N. Petrov, National Medical Research Center of Oncology named after N.N. Blokhin, National Medical Research Center for Radiology, Ministry of Health of the Russian Federation, First Saint Petersburg State Medical University named after acad. I.P. Pavlov. Local ethics committees and patient organizations are also represented among the participants. The information system will improve the quality of clinical trials, ensure the availability and security of data, reduce the production time of modern anticancer drugs, and increase the level of technological equipment of the industry as a whole. Validation of the system will be carried out in accordance with international standards. [17].

Foreign researchers also note the possible positive impact of the use of blockchain technology in medicine, healthcare, genomics, which is confirmed by a significant number of scientific studies devoted to this topic.

According to some authors, blockchain technology has become a suitable and modern solution for the secure storage and exchange of genomic data [18]. According to some studies, the market for the application of blockchain technology in the genomic market can grow at an average annual rate of $66.42 \%$ for the period 20192029 [19]. Some authors suggest the possibility of using blockchain technology to create reliable systems for storing and processing genetic information [8]. A number of authors propose to use blockchain technology to improve systems of interaction with consumers within the framework of providing services for specific people genome sequencing [13]. Very often the issues of using blockchain technology to ensure the safety and reliable transfer of personal genomic data are considered. Research on the use of blockchain technology in the circulation, processing, storage and transmission of genetic data is quite common [20].

Some authors are considering the possibility of using blockchain technology to control the exchange of genomic information [21]. Some researchers suggest using blockchain technologies for direct study and modeling of biological mechanisms, in particular, blockchain-based retrospective lineage tracing and monitoring multiplexed biochemical circuits are proposed [22]. 
As we can see, blockchain technology, due to its truly unique characteristics, is considered by many researchers as an integral part of the system for the proper functioning of genomic research and even as a basis for modeling biological processes.

At the same time, according to the authors, blockchain technology is more universal and, due to its characteristics, can be used as an integral part of the mechanism of legal regulation of genomic research, as a means, the use of which, along with other methods and techniques, can allow to ensure a balance of private, group and public interests in the field of legal regulation of genomic research. It is also necessary to note the possible positive effect of the use of blockchain technology for government in the field of genomic research in general, to develop such a methodology for resolving ethical and other contradictions, which will allow in dynamics to make meaningful decisions in the field of genomic research. In addition, the use of blockchain technology in the future may help to resolve controversial issues related to genomic research in a pretrial manner.

\section{The Balance of Interests}

It seems important to dwell a little on such an issue as ensuring a balance of interests as the basis of legal regulation in the designated area.

Recently, it is often possible to meet the opposition of human life and health, on the one hand, and the interests of science and society, on the other. Meanwhile, in our opinion, such an opposition leads to a negative effect in any case, no matter which of the interests is put at the forefront. It seems that ensuring a balance of interests, both legal and organizational, can be considered as the basis for legal regulation in this area, so we would like to dwell a little on this issue.

Interest is defined as something objectively significant, necessary for an individual, collective, society, etc. [23]. At the same time, interest in law serves as its most important law-forming and law-implementing factor [24].

With regard to the area under consideration, three levels of interests can be distinguished according to the degree of their commonality - these are private interests, group interests and general or, in other words, public interests.

Among the private interests, first of all, it is necessary to note the interests of specific people, individuals who, within the framework of the social relations under consideration, can act in various qualities - a patient, a participant in scientific research, etc. It seems that the main, legally significant direction, vector of private interests is the provision of individual rights and freedoms of individuals.

In relation to group interests, the interests of various social groups can be named as subjects. In the area under consideration, these include, for example, the interests of business, entrepreneurs, incl. large medical and / or pharmaceutical corporations, which are characterized by an independent focus, primarily on maximizing profit and often come into conflict with the interests of individuals and even with public interests.

Public interests can be attributed to the state, the purpose of which should be, first of all, to ensure the stability and security of the development of society, to maintain social consensus and balance, which determines the direction, vector of interest in this case.

Thus, it is obvious that there are at least three levels of interests, which often come into conflict with each other and need to find a balance.

It seems that when considering approaches to determine the balance of interests in this case, it is necessary to take into account the following factors.

1. The dynamic development of the regulated sphere of social relations, due to the advancement of technologies in the field of genomic research in recent years.

2. Extreme sensitivity of the relevant sphere of social relations, including in relation to a specific person, especially when it comes to genomic research in the field of human reproduction.

3. A significant number of relations between subjects of different states in the considered sphere of social relations.

4. Close connection of the sphere in question with human rights law as an independent branch of international law.

5. Significant influence of ethics and morality on social relations in this area.

Taking into account these factors, it can be assumed that in order to ensure a balance of private, group and general (public) interests, it is necessary to establish sufficiently clear norms in positive law, including norms at the level of international law and the law of interstate integration formations. Also it is necessary to use a symbiotic regulator, including both legal norms and norms of another social nature, different from legal ones. In addition, there is a need for a credible and reliable mechanism that would make it possible to resolve emerging issues and contradictions of both ethical and legal nature.

\section{A mechanism to ensure a balance of interests}

As for the mechanism for maintaining a balance of private, group and general (public) interests in the legal regulation of genomic research, it seems that this mechanism consist of three main elements:

- normative regulation (primarily developed, wellresearched legal regulation in the relevant area);

- the presence of effectively functioning organizational structures, the purpose of which will be to solve specific tactical and strategic issues of the development and functioning of the relevant industry;

- properly working processes of interaction of organizational structures and subjects of relations in the considered area of social relations.

Let us take a closer look at each of these elements. 
In terms of normative regulation, we are talking not only about legal aspects, but also about regulation carried out with the use of other social regulators.

Thus, legal regulation is an effective, regulatory and organizational impact on social relations carried out using a system of legal means (legal norms, legal relations, individual prescriptions, etc.) in order to streamline them, protect and develop in accordance with social needs [25].

Meanwhile, within the framework of normative regulation as an element of the mechanism for ensuring the balance of private, group and public interests, it is necessary to associate normative regulation not only with the legal norms per se, but also with the norms of morality, ethics, and other social regulators.

The second element of the mechanism that characterizes the statics of the corresponding social relations is the presence of effectively functioning organizational structures. We are talking here about structures associated with public authorities, for example, state bodies, services, agencies, etc., and selfregulatory bodies, for example, public organizations, ethical committees, patient associations, communities of medical workers. Moreover, it seems that in this sphere of social relations, maintaining a balance is possible if there are well-developed and competent self-regulation structures. They will be able to play an important role in ensuring the balance of private, group and public interests.

The third element that characterizes the dynamics of the relevant social relations is the properly functioning processes of interaction between bodies and subjects within the framework of the designated sphere of social relations. Note that it is possible to speak about the proper functioning only when the process, with the same initial data, leads to the same result. In relation to the field of genomic research, we can also say that the corresponding processes should proceed in a protected mode, in compliance with safety requirements, as well as the safety of the relevant information during its storage, transmission, exchange and other processing methods.

\section{Blockchain technology as an element of a balance mechanism}

As shown above, in order to ensure a balance of interests, a credible and reliable mechanism is needed that would make it possible to promptly resolve emerging issues and contradictions of both ethical and legal nature.

Blockchain technology, as having unique characteristics, can be an important basic element of the mechanism for maintaining a balance, and this technology can be applied in each of the parts of this mechanism, both within the framework of normative regulation, within the framework of public administration and within the framework of interaction between subjects of social relations.

The blockchain technology can also be used as a kind of trusted environment within which the corresponding processes are carried out and conducted, for example, information is exchanged, or a question is discussed, and certain data is stored. Blockchain technology can also be used as a kind of means, a tool for ensuring the security and safety of information exchange within the framework of a mechanism to ensure a balance of interests. Blockchain technology and the possibility of using the so-called smart contracts embedded in this technology, which are often defined as sets of instructions that are applied subject to certain conditions, the authenticity, conditions and needs of which can be met and approved by everyone [8], can be used as methodology for resolving certain issues arising in the course of genomic research. In addition, by using blockchain technology it is possible to develop information systems for resolving disputes and contradictions arising between the subjects of relevant relations, which will also help to ensure a balance of interests in this area.

\section{Conclusion}

Based on the research carried out, the following conclusions can be drawn. Blockchain technology is used in practice to ensure the safety and security of sensitive information, such as personal genomic information, medical data. The technology is also considered by many researchers as promising enough for the use in various fields of genomic research.

Blockchain technology is universal and, due to its characteristics, can be used as an integral part of the mechanism of legal regulation of genomic research, as a means that, along with other methods and techniques, can provide a balance of private, group and public interests in the field of legal regulation of genomic research. A positive effect is also possible from the use of blockchain technology for public administration in the field of genomic research in general, for the development of such a methodology for resolving ethical and other contradictions, which will allow making significant decisions in the field of genomic research in dynamics. The use of blockchain technology in the future may become the basis for creating a platform that allows real-time pre-trial solution of controversial issues related to genomic research.

To ensure a balance of private, group and general interests, along with the need to establish clear and welldeveloped norms in positive law, including at the level of international law and the law of interstate integration formations, it seems possible to use a symbiotic regulator, including both legal norms and norms of other social nature. As an element of ensuring a balance of interests, a credible and reliable mechanism is needed to promptly resolve emerging issues and contradictions of both ethical and legal nature. A balance-of-interest mechanism consist of three main elements:

- normative regulation (primarily developed, wellresearched legal regulation in the relevant area);

the presence of effectively functioning organizational structures, the purpose of which will be to solve specific tactical and strategic issues of the development and functioning of the relevant industry; 
- properly working processes of interaction of organizational structures and subjects of relations in the considered area of public relations.

Blockchain technology can be an important basic element of the balance mechanism that can be applied to each of the parts of the specified mechanism. Blockchain technology can be used as a medium for the flow of processes, as a means, a tool within the framework of the mechanism for ensuring a balance, and also as a method for resolving issues arising in the framework of relevant social relations.

This article is based on the results obtained within the framework of the state assignment of the Ministry of Education and Science of Russia 730000Ф.99.1.БB16AA02001 ('Scientific and methodological support', Kutafin Moscow State Law University), the Russian Foundation for Basic Research grants 18-29-14078 MK and 18-29-14054 MK.

\section{References}

1. Decree of the President of the Russian Federation of 09.05.2017 N 203 "On the Strategy for the Development of the Information Society in the Russian Federation for 2017 - 2030". "Collected Legislation of the Russian Federation", 05.15.2017, N 20, art. 2901.

2. B.A. Raizberg, L.Sh. Lozovsky, E.B. Starodubtseva, Modern Dictionary of Economics, 6th ed. (INFRAM, Moscow, 2011)

3. D. Bell, The coming post-industrial society: the experience of social forecasting (Academia, Moscow, 1999)

4. A.T. Tufetulov, Information economy and information society, Actual problems of economics and law (2017)

5. I. Lazarev, K. Lazarev, G. Khizha, New information economy and network mechanisms of its development, Economic strategies, 8 (2005)

6. V.V. Fedotova, B.G. Emelyanov, L.M. Tipner, The concept of blockchain and the possibilities of its use, European Science, 1(33) (2018)

7. V.B. Nagrodskaya, New technologies (blockchain / artificial intelligence) in the service of law: scientific and methodological manual (L.A. Novoselova, ed.) (Prospect, Moscow, 2019)

8. Halil Ibrahim Ozercan, Atalay Mert Ileri, Erman Ayday, Can Alkan, Realizing the potential of blockchain technologies in genomics, Genome Res., 28, 1255-1263 (2018)

9. P. Zhang, J. White, D.C. Schmidt, G. Lenz, S.T. Rosenbloom, FHIRChain: applying blockchain to securely and scalably share clinical data, Comput. Struct. Biotechnol. J., 16, 267-78 (2018). doi: 10.1016 / j.csbj.2018.07.004. https://linkinghub.elsevier.com/retrieve/pii/S20010370(18)30037-0.

10. G. Chris, D. Alos, Increasing Supply Chain Assurance via the Blockchain (2016). Retrieved from:

https://www.academia.edu/19623721/Increasing_Su pply_Chain_Assurance_via_the_Blockchain

11. M. Holbl, M. Kompara, A. Kamlsalic, L.N. Zlatolas, A systematic review of the use of blockchain in Healthcare, Symmetry-Basel, 10, 470 (2018). doi: 10.3390 / sym10100470. doi: 10.3390 / sym10100470.

12. G. Paul, P. Sarkar, S. Mukherjee, Towards a More Democratic Mining in Bitcoins, Proceedings of the International Conference on Information Systems Security; ICISS'14 (December 16-17, 2014; Hong Kong) pp. 185-203.

13. Xiao-Ling Jin, Miao Zhang, Zhongyun Zhou, Xiaoyu $\mathrm{Yu}$, Application of a Blockchain Platform to Manage and Secure Personal Genomic Data: A Case Study of LifeCODE.ai in China, J. Med. Internet Res., 21(9), 13587 (2019)

14. Retrieved from: https: //digiforest.io/blog/blockchain-examples-inhealthcare accessed 06/27/2021

15. Retrieved from: https: //www.securingindustry.com/pharmaceuticals/mong olia-to-pilot-national-blockchain-drug-traceabilitysystem/s40/a8869/\#.X1aR33kzZPZ accessed $06 / 27 / 2021$

16. Retrieved from: https: //tass.ru/ekonomika/5123036 date of treatment 06/27/2021

17. A.M. Belyaev, I.S. Ogilidi, A.D. Caprin, M.R. Lichinitser, A.A. Meshcheryakov, V.F. Semiglazov, E.N. Imyanitov, T.Yu. Semiglazova, A.N. Poltoratsky, L.V. Konstantinov, I.A. Petrusenko, O. I. Nikitin, K.A. Zakharov, M.I. Trifonov, D.N. Plakhov, Blockchain in healthcare: opportunities for use in clinical trials, General Medicine, 2, 2018

18. Sami Ullah, S. Aslam, N. Arjomand, Blockchain in Healthcare and Medicine: A Contemporary Research of Applications, Challenges, and Future Perspectives, retrieved from: https://arxiv.org/abs/2004.06795, last visit 06/27/2021

19. R. and Markets, Blockchain in Genomics | Global Market Analysis Through 2019-2029, Projecting a CAGR of $66.42 \%$, Globe Newswire News Room, (Oct. 22, 2019). Retrieved from: http://www.globenewswire.com/newsrelease/2019/10/22/1933431/0/en/Blockchain-inGenomicsGlobal-Market-Analysis-Through-20192029-Projecting-a- CAGR-of-66-42.html (last visit 06/27/2021).

20. R. Carlini, F. Carlini, S. Dalla Palma, R. Pareschi, Genesy: a Blockchain-based Platform for DNA Sequencing. Retrieved from: http://ceur-ws.org/Vol2334/DLTpaper6.pdf

21. Mahsa Shabani, Blockchain-based platforms for genomic data sharing: a de-centralized approach in response to the governance problems? Journal of the 
American Medical Informatics Association, 26(1), 76-80 (2019)

22. A. C. Chin, Blockchain Biology, Front. Blockchain (01 December 2020). DOI: https://doi.org/10.3389/fbloc.2020.606413

23. Philosophical Dictionary (Moscow, 1980) p. 131.

24. N.N. Kosarenko, Category "interest" in the system of public and private law, Knowledge. Understanding. Skill, 3, 157 (2007)

25. S.S. Alekseev, Theory of law (Publishing house BEK, Moscow, 1995) p. 209. 